\title{
Melioidosis case cluster following heavy inter-monsoonal rains in the Eastern Province of Sri Lanka
}

\author{
VRFrancis ${ }^{1}$, MAhilen ${ }^{2}$, MMurugamoorthy $^{2}$, KArulmoly ${ }^{2}$, KTSundaresan $^{2}$, \\ WASKWickramarachchi ${ }^{2}$, PMayurathan ${ }^{2}$, HSathkumara $^{3}$, SKrishnananthasivam $^{3}$, JMasakorala ${ }^{4}$, \\ ADDe Silva ${ }^{3}$, EMCorea $^{4}$
}

\section{Introduction}

Melioidosis is an emerging tropical infection caused by the soil bacterium Burkholderiapseudomallei. Sri Lanka has recently been identified as endemic for the disease. The study describes a cluster of cases of acute bacteraemic melioidosis following an extreme weather event. This study describes the clinical, epidemiological and bacteriological characteristics of a cluster of bacteraemic melioidosis cases.

\section{Methods}

Cultures from patients were referred to the reference laboratory and confirmed by real time polymerase chain reaction (RT-PCR). Clinical histories were recorded. Multi locus sequence typing was performed for genotyping. Rainfall data in Batticaloa for October 2015 was obtained from the Department of Meteorology of Sri Lanka.

\section{Results}

Ten culture positive, adult cases of melioidosis were identified in the Batticaloa District of the Eastern Province of Sri Lanka between November and December 2015, following heavy rainfall in October. All had direct or indirect exposure to flood water. Only two were farmers. Six had diabetes mellitus. All ten cases presented with fever and bacteraemia. Focus of infection was identified in 8 patients (pneumonia - 3, sinusitis - 2, joint infections - 2 and pyelonephritis -1). Four patients ( 3 pneumonia and one with pyelonephritis) died, giving a case fatality rate of $40 \%$, which is significantly higher than the case fatality rate of $23 \%$ recorded in sporadic cases. Although two had co-morbidities, two were healthy young females who presented with pneumonia, septic arthritis (indicating dissemination) and septicaemia and had a severe acute course. Four isolates were YLF while 6 isolates belong to the BTFC gene cluster which is uncommon in Asia, with four isolates being of a single multilocus sequence type (ST594).

\section{Discussion and Conclusion}

A single ST (ST594) was responsible for a significant proportion (40\%) of cases. The reason for this is unclear. Inadequate community-based detection and control of diabetes was the major underlying factor. Late presentation, poor control of diabetes and late recognition contributed to the high mortality. Primary prevention of melioidosis is difficult because of the saprophytic nature of the organism and the inevitability of flooding during the rainy season. Secondary

\footnotetext{
${ }^{1}$ Faculty of Healthcare Sciences, Eastern University of Sri Lanka

${ }^{2}$ Teaching Hospital, Batticaloa, Sri Lanka

${ }^{3}$ Genetech Research Institute, Colombo 8, Sri Lanka

${ }^{4}$ Department of Microbiology, Faculty of Medicine, University of Colombo, Sri Lanka

Address for correspondence: Dr Vaithehi Francis, Department of Microbiology, Faculty of Healthcare Sciences, Eastern University of Sri Lanka. +94 773642462 Email: rajeevanf@yahoo.com 1 https://orcid.org/ 0000-00031854-0796
} 
prevention, by early suspicion and culture confirmation, requires close coordination between clinicians and microbiologists. 